\title{
Positive effects of Nordic Walking on anthropometric and metabolic variables in women with type 2 diabetes mellitus
}

\section{Effets positifs de la Marche Nordique sur les variables anthropométriques et métaboliques chez les femmes atteintes de diabète de type 2}

\author{
F. Sentinelli $i^{a, b}$, V. La Cava ${ }^{b}$, R. Serpe ${ }^{b}, A$. Boi $^{b}$, M. Incani ${ }^{b}$, \\ E. Manconi ${ }^{b}$, A. Solinas ${ }^{b}$, E. Cossu ${ }^{b}$, A. Lenzi ${ }^{a}$, M.G. Baroni ${ }^{a, *, b}$ \\ a Department of Experimental Medicine, Sapienza University of Rome, Policlinico Umberto I, 00161 Rome, \\ Italy \\ ${ }^{\mathrm{b}}$ Department of Medical Sciences, University of Cagliari, Cagliari, Italy
}

Received 25 June 2014; accepted 16 October 2014

Available online 20 November 2014

\section{KEYWORDS}

Nordic Walking;

$\mathrm{HbA}_{1 \mathrm{c}}$;

$\mathrm{BMl}$;

Obesity;

Physical activity; Weight reduction

\begin{abstract}
Summary
Objectives. - Lack of physical activity predisposes people to chronic diseases including diabetes mellitus, obesity, and coronary artery diseases. Identifying forms of physical activity is warranted for prevention of these chronic diseases. Daily exercise has also been considered a significant contributing factor in the management of type 2 diabetes. Nordic Walking is shown to be easy to teach and learn, simple and adaptable to subjects with diabetes and metabolic syndrome. Nordic Walking allows training of about $90 \%$ of body muscles in the active propulsion phase, thus increasing energy expenditure by 30 to $50 \%$. Aim of our study was to evaluate the effects of Nordic Walking structured training in a group of female patients with type 2 diabetes, looking at changes in anthropometric, metabolic and bioelectrical variables pre- and post-activities. A follow-up of 6 months after the end of the program was also performed. Equipment and methods. - Twenty women with type 2 diabetes, aged 40-65years, were enrolled. The participants were randomized in two groups: (1) 10 women in the Nordic Walking active group, (2) 10 women receiving counseling alone to perform physical activity (control group).
\end{abstract}

\footnotetext{
* Corresponding author.

E-mail address: marco.baroni@uniroma1.it (M.G. Baroni).
} 
Results. - Results show that diabetic women involved in a 12-week structured Nordic Walking program obtain a significant reduction in $\mathrm{HbA}_{1 \mathrm{c}}(-0.7 \%)$, BMI $\left(-0.8 \mathrm{~kg} \cdot \mathrm{m}^{-2}\right)$ and body weight $(-2.4 \mathrm{~kg})$, and a significant increase in $\mathrm{HDL}$ cholesterol $\left(+5.8 \mathrm{mg} \cdot \mathrm{dL}^{-1}\right)$. Also Handgrip strength was significantly improved $(+4.3 \mathrm{~kg})$. At follow-up all changes returned to baseline values.

Conclusions. - Our study shows that a supervised Nordic Walking program determines a significant improvement in metabolic and anthropometric parameters in women with type 2 diabetes. (c) 2014 Elsevier Masson SAS. All rights reserved.

\section{MOTS CLÉS}

Marche nordique ;

$\mathrm{HbA}_{1 \mathrm{c}}$;

L'IMC ;

Obésité ;

Activité physique ;

Réduction de poids

\section{Background}

Modern lifestyle is characterized by the lack of everyday physical activity and exercise training, predisposing people to chronic diseases including diabetes mellitus, obesity, hypertension, and coronary artery diseases [1]. Therefore, identifying forms of physical activity that are easily accessible and can be performed by a large number of people is warranted to prevent or reduce the incidence of these chronic diseases.

Daily exercise has been considered a significant contributing factor in the management of type 2 diabetes as indicated by several studies [2-4]. Prospective cohort studies in subjects with or without T2DM [5,6] have provided evidence for significant positive effects in terms of prevention of T2DM, improved metabolic control and reduced mortality in the physically active compared to sedentary individuals. For example, regular walking increases insulin sensitivity and reduces cardiovascular risk factors such as hypertension [7], dyslipidemia [8] and fat mass accumulation [9]. Several studies have investigated the effects of aerobic, resistance and combined aerobic and resistance training in adults with type 2 diabetes [4,10-12]; all showed beneficial effects in metabolic control, body weight, insulin resistance, fat distribution, with some differences in aerobic vs. resistance training. Also most studies showed that supervised training was more effective than simple counseling $[13,14]$.

Nordic Walking, developed in Scandinavia and introduced in central Europe nearly 20 years ago, is a fitness type of walking which also activates the muscles of the upper limbs with the use of specially designed walking poles. Previous studies have documented that the additional activity of the arms increases oxygen consumption, caloric expenditure and heart rate, with beneficial effects that may reduce cardiovascular risk and improve metabolic control $[15,16]$.

Nordic Walking has been proven to be a simple and feasible form of physical activity that can be performed by nearly everybody, everywhere, and at almost any time. It has the characteristic, therefore, to have the appropriate intensity and simplicity to induce fitness and health.

The aim of this study was to evaluate the effects of Nordic Walking structured training in a group of female patients 
with type 2 diabetes, looking at changes in anthropometric, metabolic and bioelectrical variables at baseline and at the end of a 12-week activity program. Furthermore, we looked at changes in anthropometric and metabolic variables after six months from the interruption of Nordic Walking supervision and guidance.

\section{Patients and methods}

\subsection{Study design and subjects}

We enrolled 20 women with type 2 diabetes in the study, aged between 40 and 65 years. The 20 participants were randomized in two groups:

- a group of 10 women in the Nordic Walking active group; - a group of 10 women with the same clinical features that have received counseling alone to perform physical activity (control group).

All participants were sedentary, and did not report physical activities in the 6 months prior to the enrollment.

The active group carried out supervised Nordic Walking for a period of 12 weeks with a frequency of 3 sessions per week for 60-90 minutes, session characterized by incremental volumes of training.

Clinical and metabolic measurements were performed at baseline (T0), 3 months from baseline (T1) and after a follow-up period of 6 months (i.e. 9 months from baseline, T2) in order to study the effects of the activities carried out by the Nordic Walking group and by the control group.

The 10 subjects of the control non-active group were advised to maintain an active lifestyle, consisting in at least 50 minutes of physical exercise (of their choice) three times per week. Thus participants were not following a specific training program, but were recommended to exercise. Examples of exercises and informative leaflets were given to all subjects in the control group.

Inclusion criteria were: diagnosis of type 2 diabetes for more than 1 year, an $\mathrm{HbA}_{1 \mathrm{c}}$ in the range of $6 \%$ to $10 \%$, body mass index $>25 \mathrm{~kg} \cdot \mathrm{m}^{-2}$, age in the range of 40 to 70 years, and stable antidiabetic treatment for atleast 3 months before inclusion. Exclusion criteria were symptomatic heart disease (NYHA 2-4), lower extremities vascular disease, myocardial infarction within the past 6 months, and severe lung disease.

A physical examination was performed before inclusion in the study. General dietary habits were recorded at baseline and at the end of the training period, in order to evaluate if substantial changes were implemented.

\subsection{Intervention and training methodology}

The training was divided into two phases of work.

In the first phase, lasting 6 weeks, the team's work has focused on the execution of exercises aimed at developing the proper technical gesture typical of Nordic Walking, including a training period of low/medium intensity aimed to evaluate fitness level of each subject and to increase their cardiovascular resistance.
In the second phase, lasting other six weeks, the patients in the active group performed NW sessions of medium/high intensity and difficulty, characterized by increasing and progressive exercise loads.

The training sessions, characterized by warm-up, conditioning phase, stretching and breathing exercises, have been adapted to the diabetic women. During warm-up, exercises for joint mobility were performed with flexion and extension of the arms, legs and trunk with the aid of sticks.

The conditioning phase saw the group initially engaged in stretches of low/medium difficulty of 4-5 km with gentle slopes (7\%), and later walks of medium/high difficulty of the length of around $7 \mathrm{~km}$ with slopes between $10 \%$ and $14 \%$.

In each phase the patients were constantly monitored by measurement of the heart of the radial pulse.

In the last part of the workout specific stretching exercises for muscle chains involved in Nordic Walking were chosen, carried out with the aid of the sticks and associated with breathing exercises to promote optimal recovery and relieve of muscle tension.

Written informed consent was obtained from all participants. The study was approved by the University's Ethical Committee.

\subsection{Clinical and metabolic variables}

For all patients whole blood and serum samples were collected for measurement of laboratory variables. $\mathrm{HbA}_{1 \mathrm{c}}$ was measured using a G8 analyzer (THOSO Diagnostics, Tokyo, Japan) by HPLC, aligned with IFCC standardization, according to DCCT/UKPDS guidelines [17].

Data regarding glucose, lipids (total cholesterol, HDL, LDL and triglycerides), aspartate aminotransferase (AST), alanine aminotransferase (ALT), were also measured by standard laboratory methods. BoDY MASS INdex (BMI) was calculated by the formula body weight $(\mathrm{kg})$, divided by height $(\mathrm{m})$ squared.

\subsection{Bioelectric impedance}

The body composition assessment was conducted through electrical impedance analysis by standard protocols, with an Akern STA/BIA 101 series analyzer (Akern, Italy). The electrical data obtained from each patient were processed by Akern Body Gram 3.1 software (Akern, Italy), obtaining the percentages of the different body compartments, defined as the percentage of lean body mass (LBM), fat-free mass (FFM), muscle mass (MM), fat mass (FM), and total body water (TBW).

\subsection{Handgrip strength}

The evaluation of the "grip strength", a functional parameter of muscle strength was measured by hydro-mechanical dynamometer (Jamar, Sammons Preston, Canada). The test was performed with the subjects squeezing the dynamometer with the dominant limb $(90 \% \mathrm{R}, 10 \% \mathrm{~L})$, evaluating the force of hand strength. The Handgrip Test has been included in the study as a method for assessing upper limb strength, as 
the physical activity proposed determined a direct involvement of the upper portion of the body.

\subsection{Statistics}

SPSS version 17 statistical package was used to perform the statistical analyses. These included Student's $t$ test for normally distributed variables, Mann-Whitney $U$ independent sample test for non-normal distributions. For paired analyses, repeated measure ANOVA was used to analyze within-group differences. Non-normally distributed variables (TG, GPT, GGT) were log-transformed before analysis.

Differences of medication and physical activity levels were analyzed by $\mathrm{Chi}^{2}$ or Fisher's exact test. A $P$ value $<0.05$ was regarded as statistically significant.

Power calculation: Data in the control group, taken as reference, indicate that $\mathrm{HbA}_{1 \mathrm{c}}$ standard deviation is $\pm 0,7$. If the true difference in the $\mathrm{HbA}_{1 \mathrm{c}}$ mean after treatment of our subjects is 0.7 , we will be able to reject the null hypothesis that this difference is zero with probability (power) $80 \%$, with a type I error probability of 0.05 .

\section{Results}

\subsection{Clinical and metabolic characteristics of the study subjects at baseline}

Anthropometric and metabolic characteristics at baseline in both studied groups are shown in Table 1 . Among the 20 participants, aged between 40 and 65 years, 50\% in the active group were obese, $20 \%$ overweight and $10 \%$ normal weight. In the control group, $50 \%$ were obese, $30 \%$ overweight and $20 \%$ normal weight. Power calculation demonstrated that this sample size had a power of $80 \%$, to detect a significant difference in $\mathrm{HbA}_{1 \mathrm{c}}$ after the training period. The $\mathrm{BMI}$ and glycosylated hemoglobin were comparable between the two groups. Also lipid profile and bioelectric impedance variables were not significantly different between the two groups. In the NW group liver parameters were higher compared to control women.

\subsection{Effect of Nordic Walking on clinical and metabolic variables}

As discussed in the methods, 10 patients followed a 12-week activity program of Nordic Walking (NW). None of the study participants suspended this activity before the end of the 12-week period.

The other 10 patients followed standard lifestyle recommendations. The standard lifestyle intervention consisted in dietary and physical activity recommendations. Briefly, to all patients dietary guidelines were proposed, recommending the adoption of a normocaloric Mediterranean diet based on a balanced distribution of carbohydrates (55\%), proteins (15\%), and lipids (30\% total, with less than $10 \%$ saturated fat). Modifications in physical activity consisted in the recommendation to perform aerobic exercise three to five times per week for at least 45-60 min.

The comparison between the anthropometric and metabolic variables at time zero and at the end of 12 weeks
Table 1 Clinical, biochemical and bioelectric variables characteristics of study population at TO.

\begin{tabular}{|c|c|c|c|}
\hline & Controls & $\begin{array}{l}\text { Nordic Walking } \\
\text { subjects }\end{array}$ & $P$ \\
\hline Age (years) & $60 \pm 5$ & $54 \pm 9$ & 0.090 \\
\hline Weight (kg) & $75.8 \pm 16.1$ & $82.5 \pm 16.1$ & 0.369 \\
\hline BMI $\left(\mathrm{kg} \cdot \mathrm{m}^{-2}\right)$ & $32 \pm 7$ & $32.3 \pm 6$ & 0.827 \\
\hline $\mathrm{FBG}\left(\mathrm{mg} \cdot \mathrm{dL}^{-1}\right)$ & $156 \pm 47$ & $136 \pm 28$ & 0.287 \\
\hline $\mathrm{HbA}_{1 \mathrm{c}}(\%)$ & $7.1 \pm 1.3$ & $7.1 \pm 1.3$ & 1.000 \\
\hline $\mathrm{TC}\left(\mathrm{mg} \cdot \mathrm{dL}^{-1}\right)$ & $199 \pm 43$ & $168 \pm 32$ & 0.084 \\
\hline $\mathrm{TG}\left(\mathrm{mg} \cdot \mathrm{dL}^{-1}\right)$ & $109 \pm 60$ & $125 \pm 47$ & 0.506 \\
\hline $\mathrm{HDL}-\mathrm{C}\left(\mathrm{mg} \cdot \mathrm{dL}^{-1}\right)$ & $56 \pm 16$ & $49.2 \pm 12$ & 0.275 \\
\hline $\mathrm{LDL}-\mathrm{C}\left(\mathrm{mg} \cdot \mathrm{dL}^{-1}\right)$ & $118 \pm 39$ & $92.2 \pm 23$ & 0.086 \\
\hline GGT (U/L) & $21 \pm 6$ & $40 \pm 29$ & 0.035 \\
\hline AST (U/L) & $19 \pm 8$ & $29 \pm 11$ & 0.035 \\
\hline ALT (U/L) & $20 \pm 7$ & $38 \pm 22$ & 0.009 \\
\hline $\begin{array}{l}\text { Diabetes duration } \\
\quad \text { (years) }\end{array}$ & $7 \pm 5$ & $6 \pm 5$ & 0.769 \\
\hline FM (kg) & $30 \pm 11$ & $33.3 \pm 13.1$ & 0.564 \\
\hline FM \% & $38.3 \pm 7.7$ & $38 \pm 11$ & 0.954 \\
\hline FFM (kg) & $45.8 \pm 5.7$ & $51 \pm 5.8$ & 0.064 \\
\hline FFM \% & $61.7 \pm 7.7$ & $62 \pm 11$ & 0.954 \\
\hline TBW (L) & $34.8 \pm 4.5$ & $38.5 \pm 4.4$ & 0.088 \\
\hline TBW \% & $46.7 \pm 4.8$ & $46.6 \pm 7.4$ & 0.976 \\
\hline Handgrip Test (kg) & $27 \pm 5$ & $27.8 \pm 3$ & 0.628 \\
\hline
\end{tabular}

Values are means \pm standard deviations. Student's $t$ test for normally distributed variables, and Mann-Whitney $U$ independent sample test for non-normal distributions, were applied. $P$ values < 0.05 are considered significant. BMI: body mass index; FBG: fasting blood glucose; $\mathrm{HbA}_{1 \mathrm{c}}$ : glycated hemoglobin A1c; TC: total cholesterol; TG: triglycerides; HDL-C: high-density lipoprotein-cholesterol; LDL-C: low-density lipoprotein; GGT: gamma-glutamyltransferase; AST: aspartate aminotransferase: ALT; alanine aminotransferase; FM: fat mass; FFM: fat-free mass; TBW: total body water; U: unit; L: liter.

of NW activity (T1) is shown in Table 2. We observed, in the NW group, a significant difference at T1 in weight, with an average reduction of $2.4 \mathrm{~kg}(P<0.008)($ Fig. $1 \mathrm{~A})$, a significant decrease in BMI $(P<0.022)$, and a significant reduction of glycosylated hemoglobin $\left(\mathrm{HbA}_{1 \mathrm{c}}\right)$ from 7.1 to $6.4(P<0.01)$ (Fig. 1B). Also lipid parameters showed an improvement, with increased HDL cholesterol $(P<0.003)$ and lower triglycerides. Finally, a significant reduction in ALT transaminases $(P<0.045)$ was observed.

Table 2 also compares the values of the bioelectric and fitness variables. We observed a reduction in fat mass (FM), although not significant, and in total body water (TBW) $(P<0.036)$. A significant increase in strength, as assessed by the Handgrip Test, from 27.8 to $32.1 \mathrm{~kg}$ was observed, with a difference of $4,3 \mathrm{~kg}(P<0.014)$.

\subsection{Effect of standard recommendations on clinical and metabolic variables in the non-active cohort}

Table 3 shows anthropometric variables and metabolic values at the two times (T0 and T1) of the study in the control 
Table 2 Clinical, biochemical and bioelectric variables of active group after follow-up.

\begin{tabular}{|c|c|c|c|c|c|c|}
\hline & TO & $\mathrm{T} 1$ & $P_{1 \text { vs } 0}$ & $\mathrm{~T} 2$ & $P_{2 v s 1}$ & $P_{2 \mathrm{vs} 0}$ \\
\hline Weight (kg) & $82.5 \pm 16.1$ & $80.1 \pm 16.4$ & 0.008 & $82.1 \pm 17$ & 0.032 & 0.309 \\
\hline BMI $\left(\mathrm{kg} \cdot \mathrm{m}^{-2}\right)$ & $32.3 \pm 6$ & $31.5 \pm 6$ & 0.022 & $32.2 \pm 6.3$ & 0.030 & 0.433 \\
\hline FBG $\left(\mathrm{mg} \cdot \mathrm{dL}^{-1}\right)$ & $136 \pm 28$ & $121 \pm 19$ & 0.009 & $131 \pm 26$ & 0.196 & 0.340 \\
\hline $\mathrm{HbA}_{1 \mathrm{c}}(\%)$ & $7.1 \pm 1.3$ & $6.4 \pm 10$ & 0.009 & $6.9 \pm 1.1$ & 0.030 & 0.628 \\
\hline $\mathrm{TC}\left(\mathrm{mg} \cdot \mathrm{dL}^{-1}\right)$ & $168 \pm 32$ & $168 \pm 23$ & 0.860 & $174.8 \pm 34$ & 0.199 & 0.195 \\
\hline $\mathrm{TG}\left(\mathrm{mg} \cdot \mathrm{dL}^{-1}\right)$ & $125 \pm 47$ & $117 \pm 51$ & 0.136 & $148.3 \pm 98.3$ & 0.350 & 0.523 \\
\hline $\mathrm{HDL}-\mathrm{C}\left(\mathrm{mg} \cdot \mathrm{dL}^{-1}\right)$ & $49.2 \pm 12$ & $55 \pm 11$ & 0.039 & $51 \pm 17$ & 0.526 & 0.433 \\
\hline LDL-C (mg.dL $\left.{ }^{-1}\right)$ & $92.2 \pm 23$ & $89 \pm 12.4$ & 0.765 & $94.5 \pm 25.3$ & 0.379 & 0.539 \\
\hline GGT (U/L) & $40 \pm 29$ & $38 \pm 26.5$ & 0.354 & $49.8 \pm 38.5$ & 0.151 & 0.324 \\
\hline AST (U/L) & $29 \pm 11$ & $27 \pm 8.1$ & 0.821 & $27 \pm 12.5$ & 0.623 & 0.368 \\
\hline ALT (U/L) & $38 \pm 22$ & $32 \pm 18.6$ & 0.045 & $36 \pm 23$ & 0.573 & 0.346 \\
\hline FM (kg) & $33.3 \pm 13.1$ & $31.8 \pm 13.1$ & 0.786 & $30 \pm 14.8$ & 0.802 & 0.925 \\
\hline FM \% & $38 \pm 11$ & $38.1 \pm 10$ & 0.536 & $36 \pm 13.1$ & 0.668 & 0.904 \\
\hline FFM (kg) & $51 \pm 5.8$ & $48.3 \pm 5.3$ & 0.193 & $48.9 \pm 5$ & 0.538 & 0.753 \\
\hline FFM \% & $62 \pm 11$ & $62 \pm 10$ & 0.536 & $64.1 \pm 13.1$ & 0.815 & 0.904 \\
\hline TBW (L) & $38.5 \pm 4.4$ & $36.3 \pm 4.1$ & 0.041 & $36.7 \pm 3.3$ & 0.537 & 0.793 \\
\hline TBW \% & $46.6 \pm 7.4$ & $46.4 \pm 7.4$ & 0.327 & $48 \pm 9.4$ & 0.810 & 0.876 \\
\hline Handgrip Test (kg) & $27.8 \pm 3$ & $32.1 \pm 4.1$ & 0.032 & $33 \pm 4.6$ & 0.042 & 0.086 \\
\hline
\end{tabular}

Values are means \pm standard deviations. For paired analyses, repeated measure ANOVA was used to analyze within-group differences. Non-normally distributed variables (TG, GPT, GGT) were log-transformed before analysis. $P$ values $<0.05$ are considered significant. BMI: body mass index; FBG: fasting blood glucose; $\mathrm{HbA}_{1 \mathrm{c}}$ : glycated hemoglobin A1c; TC: total cholesterol; TG: triglycerides; HDL-C: high-density lipoprotein-cholesterol; LDL-C: low-density lipoprotein; GGT: gamma-glutamyltransferase; AST: aspartate aminotransferase: ALT; alanine aminotransferase; FM: fat mass; FFM: fat-free mass; TBW: total body water; U: unit; L: liter.

non-active group. The 10 subjects of this group were advised to maintain an active lifestyle and a constant practice of physical exercise.

The analysis of the data shows a significant decrease of weight of $1 \mathrm{~kg}(P<0.04)$ at the end of the 12 weeks of observation, and no significant change in all other metabolic variables. Also, no significant differences were observed in the bioelectric and fitness variables of the control group at the end of the protocol (data not shown).
We further compared the control group and the intervention group at the end of the 12 weeks of observation and the variables that were significantly different between the two cohorts were respectively: $\mathrm{HbA}_{1 \mathrm{c}}$ (7.2 \pm 1.4 vs. $6.4 \pm 1 ; P>0.02)$, total cholesterol ( $200 \pm 43$ vs. $168 \pm 23$; $P>0.035)$, LDL cholesterol $(123 \pm 39$ vs. $89 \pm 12.4 ; P>0.019$, AST $(20 \pm 9$ vs. $27 \pm 8.1 ; P>0.043)$, and Handgrip Test $(26.7 \pm 4.5$ vs. $32.1 \pm 4.1 ; P<0.029)$. All the other variables did not show a statistical difference.
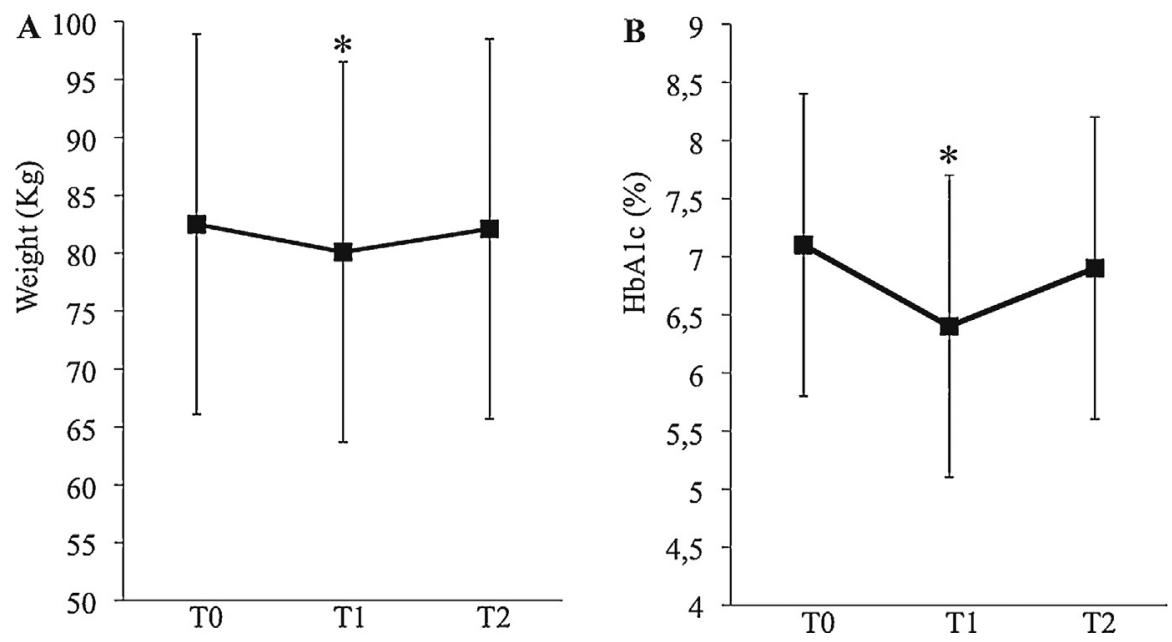

Figure 1 Weight and glycosylated hemoglobin measurements during structured training. The study cohort followed a 12-week activity program of Nordic Walking (NW), and anthropometric and metabolic variables were measured at time zero (T0), at the end of the 12 weeks of NW activity (T1) and after 6 months (T2) from the end of the activity. (A) Significant reduction in weight at time $\mathrm{T} 1(P<0.008)$. (B) Significant reduction of glycosylated hemoglobin $\left(\mathrm{HbA}_{1 \mathrm{C}}\right)$ at time $\mathrm{T} 1(P<0.01)$. 
Table 3 Clinical and biochemical variables of control group at the two times of the study.

\begin{tabular}{|c|c|c|c|}
\hline & TO & $\mathrm{T} 1$ & $P$ \\
\hline Weight (kg) & $75.8 \pm 16.1$ & $74.8 \pm 15.4$ & 0.044 \\
\hline BMI $\left(\mathrm{kg} \cdot \mathrm{m}^{-2}\right)$ & $32 \pm 7$ & $31 \pm 6$ & 0.193 \\
\hline $\mathrm{FBG}\left(\mathrm{mg} \cdot \mathrm{dL}^{-1}\right)$ & $156 \pm 47$ & $130 \pm 18$ & 0.376 \\
\hline $\mathrm{HbA}_{1 \mathrm{c}}(\%)$ & $7.1 \pm 1.3$ & $7.2 \pm 1.4$ & 0.780 \\
\hline $\mathrm{TC}\left(\mathrm{mg} \cdot \mathrm{dL}^{-1}\right)$ & $199 \pm 43$ & $200 \pm 43$ & 0.744 \\
\hline $\mathrm{TG}\left(\mathrm{mg} \cdot \mathrm{dL}^{-1}\right)$ & $109 \pm 60$ & $106 \pm 65$ & 0.707 \\
\hline $\mathrm{HDL}-\mathrm{C}\left(\mathrm{mg} \cdot \mathrm{dL}^{-1}\right)$ & $56 \pm 16$ & $56 \pm 12$ & 1.000 \\
\hline LDL-C (mg.dL $\left.{ }^{-1}\right)$ & $118 \pm 39$ & $123 \pm 39$ & 0.428 \\
\hline GGT (U/L) & $21 \pm 6$ & $24 \pm 10$ & 0.164 \\
\hline AST (U/L) & $19 \pm 8$ & $20 \pm 9$ & 0.736 \\
\hline $\operatorname{ALT}(\mathrm{U} / \mathrm{L})$ & $20 \pm 7$ & $23 \pm 9$ & 0.417 \\
\hline
\end{tabular}

Values are means \pm standard deviations. For paired analyses, repeated measure ANOVA was used to analyze withingroup differences. Non-normally distributed variables were log-transformed before analysis. $P$ values $<0.05$ are considered significant. BMI: body mass index; FBG: fasting blood glucose; $\mathrm{HbA}_{1 \mathrm{c}}$ : glycated hemoglobin $\mathrm{A} 1 \mathrm{c}$; $\mathrm{TC}$ : total cholesterol; TG: triglycerides; HDL-C: high-density lipoprotein-cholesterol; LDL-C: low-density lipoprotein; GGT: gamma-glutamyltransferase; AST: aspartate aminotransferase: ALT; alanine aminotransferase; FM: fat mass; FFM: fat-free mass; TBW: total body water; U: unit; L: liter.

\subsection{Clinical and anthropometric variables after 6 months from the end of the NW program}

All the participants to the NW program were recalled after 6 months (T2) from the end of the activity, to evaluate possible changes in clinical and metabolic variables. As shown in Table 2 and Fig. 1, weight, BMI and $\mathrm{HbA}_{1 \mathrm{c}}$ significantly worsened at T2 compared to the end of the physical activity program (T1). Also, all other lipid parameters increased compared to the time point at the end of the NW program, although not significantly. No changes were observed in the bioelectric impendence parameters.

Finally, all variables returned to values similar to those observed at baseline (T0) in the active group.

\subsection{Diabetes therapy}

With regards to therapy, $7 / 10$ of the patients in the active group were taking metformin. One was taking DPP4 (dipeptidyl peptidase-4) inhibitor, 1 a TZD (thiazolidinedione). Two patients in the NW group were on multiple daily injections (MDI) of insulin, and only 1 was taking sulfonylureas with basal insulin. These 2 patients on MDI reported episodes of hypoglycemia (one 3/weekly and the other 2 /monthly) before the start of the study. After the 3 months of physical activity only one patient changed her therapeutic regimen in the NW group, increasing metformin's dosage. In the control group 4 patients changed their therapeutic regimen, two increasing the dosage of their current therapy, one adding a GLP1-R (glucagon-like peptide-1 receptors) agonist and one repaglinide. The two patients in the active group in MDI reporting hypoglycemia had a reduction in their insulin daily dosage, and showed a great reduction in the number of episodes that were absent in one and reduced to 1 / weekly in the other.

After 6 months from the end of the supervised physical activity, 3 patients in the NW group had to change their diabetic treatment, increasing dosages of metformin or insulin, or starting a GLP1-RA. During this period no changes in the frequency of hypoglycemic episodes was reported.

\section{Discussion}

In this study, we show that type 2 diabetes women involved in a 12-week structured exercise program based on the Nordic Walking technique obtain a significant reduction in $\mathrm{HbA}_{1 \mathrm{c}}(-0.7 \%)$, BMI $\left(-0.8 \mathrm{~kg} \cdot \mathrm{m}^{-2}\right)$ and body weight $(-2.4 \mathrm{~kg})$, and a significant increase in $\mathrm{HDL}$ cholesterol $\left(+5.8 \mathrm{mg} \cdot \mathrm{dL}^{-1}\right)$. Also strength was significantly improved, as shown by the Handgrip Test $(+4.3 \mathrm{~kg})$. All these variables represent major cardiovascular (CVD) risk factors, and their reduction may reduce CVD risk in these subjects. However, these women, once interrupted the supervised NW training, returned to baseline for all variable, increasing $\mathrm{BMI}$, body weight and $\mathrm{HbA}_{1 \mathrm{c}}$.

Nordic Walking is a form of structured physical activity that involves using a pair of particular poles, similar to cross-country ski poles, in regular walking, determining the involvement of shoulder girdle, trunk and abdomen muscles. NW technique differs from normal walking, allowing training of about $90 \%$ of body muscles in the active propulsion phase, thus increasing energy expenditure by 30 to $50 \%$, and intensifying the specific work of top and middle body and improving spinal column flexibility and mobility. In particular, using poles with proper technique results in rotation of the trunk and shoulders, as well as important involvement of arms and shoulder muscles. The correct shoulder girdle rotation in NW technique directly involves many muscles groups. Postural muscles (upper trapezius, pectoralis major), spinal column stabilizers muscles (multifidus, quadrates lumborum, latissimusdorsi), abdominal muscles such as external and internal abdominal oblique and transverses abdominis are actively involved in this training.

The dynamic differences between normal walk and NW, which provides a more pronounced use of the abdominal muscles and lower back, may results in abdominal fat reserves mobilization and utilization. This may be one of the possible explanations on the significant improvement in glucose control and in body weight that we observed in our study [18].

Nordic Walking is shown to be easy to teach and learn, simple and adaptable to subjects with diabetes and metabolic syndrome, often elderly, and also with osteoarticular comorbidities [19]. As for other structured physicals programs that have been shown to be effective in counteracting metabolic parameters (i.e. glycemic control, $\mathrm{HbA}_{1 \mathrm{c}}$, decrease of body weight and adiposity, etc), NW needs a structured context (instructors). As a possible confirmation of this point is the study of Fritz et al. [20], who showed that type 2 diabetes patients improved anthropometric measurements and exercise capacity with unsupervised NW, but did not obtain a sufficient increase in exercise intensity to achieve ultimate health-promoting benefits on the 
cardiovascular parameters assessed in their study, particularly in those with altered glucose regulation.

Although in our study the NW training program was highly successful, most patients reduced or interrupted their NW activity once they completed the supervised training stage, worsening their metabolic and clinical parameters. Only 2 patients maintained their NW exercise program, and were the two with the lowest $\mathrm{HbA}_{1 \mathrm{c}}$ at the final follow-up. It may be speculated that, even for a simple exercise activity such as NW, patients may not be able to continue regularly with out programmed recalls. Furthermore, maintenance of regular physical activity is, in general, a complex task to achieve, particularly in chronic diseases. This is a confirmation of previous studies that also observed a worsening of glycemic control after the supervised program was completed $[13,14]$.

Only one randomized controlled trial (RCT) [21] examined the effects of NW in patients with type 2 diabetes mellitus. The NW group $(n=22)$ trained for 2 months ( 45 minutes, twice per week), followed by an additional 2 months (45 minutes, once per week). Results were compared to a sedentary control group $(n=22)$ and a second exercise group $(n=24)$ performing 30 minutes per week of unsupervised endurance training for 4 months. At variance with our study, there was no difference between groups with regard to $\mathrm{HbA}_{1 \mathrm{c}}$ and energy expenditure, but fat tissue mass decreased $(P=0.021)$. It must be pointed out that exercise intensity and frequency were higher in our cohort ( 3 times weekly at moderate/high intensity), thus it may be necessary to exercise at more intensity to obtain significant metabolic changes.

The NW activity had positive effects on diabetes therapy, in particular in the patients taking insulin. They had a reduction in their insulin dose, and also in the number of hypoglycemic episodes.

Our study has some limitations: first, it was performed only in women. This choice was due to the necessity to have a homogeneous group of type 2 diabetes subjects, and also to the observation that women are much more willing to exercise in group-activities. Second, we did not precisely estimate daily calorie intake. The increase in physical activity by the study participants may have changed their daily food intake to some degree, but in general our patients did not report major changes in their diet. Another limitation of our study is that we did not provide behavior change techniques (BCTs) to increase the likelihood of maintaining changes in patients' health, as shown by previous studies $[14,22]$.

Strengths of our study, on the other end, are the fact that none of the women enrolled in the active group has interrupted the program, and most participated to each session. Also the frequency of supervised weekly activity ( 3 times/week) may have been successful in determining the positive changes.

In conclusion, our study shows that a supervised NW program determines a significant improvement in metabolic and anthropometric parameters in women with type 2 diabetes. NW, by its involvement of both upper and lower extremities, may have beneficial and additional effects due to an increase in energy expenditure. Because of its simplicity and wide applicability, NW may have advantages over other supervised training programs, although continuity in supervision is necessary.

\section{Disclosure of interest}

The authors declare that they have no conflicts of interest concerning this article.

Fundings: this work was funded by research grants from the Ministry of Education, University and Research (Grant PRIN 2010-11, (number 2010JS3PMZ_006), from the Regione Autonoma della Sardegna (Grant RAS 2007 number CRP-59453), and from the Foundation Banco di Sardegna (Research Projects 2011), all awarded to Marco G. Baroni.

\section{References}

[1] Chakravarthy MV, Booth FW. Eating, exercise, and "thrifty" genotypes: connecting the dots toward an evolutionary understanding of modern chronic diseases. J Appl Physiol 2004;96:3-10.

[2] Boulé NG, Haddad E, Kenny GP, Wells GA, Sigal RJ. Effects of exercise on glycaemic control and body mass in type 2 diabetes mellitus: a meta-analysis of controlled clinical trials. JAMA 2001;286:1218-27.

[3] Snowling NJ, Hopkins WG. Effects of different modes of exercise training on glucose control and risk factors for complications in type 2 diabetic patients. A meta-analysis. Diabetes Care 2006;29:2518-27.

[4] Sigal RJ, Kenny GP, BouleŃG, Wells GA, Prud'homme D, Fortier $M$, et al. Effects of aerobic training, resistance training, or both on glycemic control in type 2 diabetes: a randomized trial. Ann Intern Med 2007;147:357-69.

[5] Smith TC, Wingard DL, Smith B, Kritz-Silverstein D, BarrettConnor E. Walking decreased risk of cardiovascular disease mortality in older adults with diabetes. J Clin Epidemiol 2007;60:309-17.

[6] Byberg L, Melhus H, Gedeborg R, Sundström J, Ahlbom A, Zethelius B, et al. Total mortality after changes in leisure time physical activity in 50 year old men: 35 year follow-up of population based cohort. Br J Sports Med 2009;43:482.

[7] Lewington S, Clarke R, Qizilbash N, Peto R, Collins R, Prospective Studies Collaboration. Age-specific relevance of usual blood pressure to vascular mortality: a meta-analysis of individual data for one million adults in 61 prospective studies. Lancet 2002;360:1903-13.

[8] Katsanos CS. Prescribing aerobic exercise for the regulation of postprandial lipid metabolism: current research and recommendations. Sports Med 2006;36:547-60.

[9] Slentz CA, Aiken LB, Houmard JA, Bales CW, Johnson JL, Tanner CJ, et al. Inactivity, exercise, and visceral fat. STRRIDE: a randomized, controlled study of exercise intensity and amount. J Appl Physiol 2005;99:1613-8.

[10] Davidson LE, Hudson R, Kilpatrick K, Kuk JL, McMillan K, Janiszewski PM, et al. Effects of exercise modality on insulin resistance and functional limitations in older adults. Arch Intern Med 2009;169:122-31.

[11] Church TS, Blair SN, Cocreham S, Johannsen N, Johnson W, Kramer $\mathrm{K}$, et al. Effects of aerobic and resistance training on hemoglobin A1c levels in patients with type 2 diabetes. JAMA 2010;304:2253-62. 
[12] Balducci S, Zanuso S, Nicolucci A, De Feo P, Cavallo S, Cardelli $P$, et al. Effect of an intensive exercise intervention strategy on modifiable cardiovascular risk factors in subjects with type 2 diabetes mellitus: a randomized controlled trial: the Italian Diabetes and Exercise Study (IDES). Arch Intern Med 2010;170:1794-803.

[13] Dunstan DW, Vulikh E, Owen N, Jolley D, Shaw J, Zimmet P. Community center-based resistance training for the maintenance of glycemic control in adults withtype 2 diabetes. Diabetes Care 2006;29:2586-91.

[14] Kirk A, Mutrie N, Maclntyre P, Fisher M. Effects of a 12-month physical counseling intervention on glycaemic control and on the status of cardiovascular risk in people with type 2 diabetes. Diabetologia 2004;47:821-32.

[15] Church TS, Earnest CP, Morss GM. Field testing of physiological responses associated with nordic walking. Res Q Exerc Sport 2002; 73:296-300.

[16] Porcari JP, Hendrickson TL, Walter PR, Terry L, Walsko G. The physiological responses to walking with and without power poles on treadmill exercise. Res Q Exerc Sport 1997;68:161-6.

[17] American Diabetes Association (ADA). Standards of medical care in diabetes. Diabetes Care 2013;36(Supp1):S11-66.
[18] Shim JM, Kwon HY, Kim HR, Kim BI, Jung JH. Comparison of the effects of walking with and without Nordic Pole on upper extremity and lower extremity muscle activation. J Phys Ther Sci 2013;25(12):1553-6.

[19] Tschentscher M, Niederseer D, Niebauer J. Health benefits of Nordic walking: a systematic review. Am J Prev Med 2013;44:76-84.

[20] Fritz T, Caidahl K, Osler M, Ostenson CG, Zierath JR, Wändell P. Effects of Nordic walking on health-related quality of life in overweight individuals with type 2 diabetes mellitus, impaired or normal glucose tolerance. Diabet Med 2011;28: 1362-72.

[21] Gram B, Christensen R, Christiansen C, Gram J. Effects of Nordic walking and exercise in type 2 diabetes mellitus: a randomized controlled trial. Clin J Sport Med 2010;20: 355-61.

[22] Hankonen N, Sutton S, Prevost AT, Simmons RK, Griffin SJ, Kinmonth $\mathrm{AL}$, et al. Which behavior change techniques are associated with changes in physical activity, diet and body mass index in people with recently diagnosed diabetes? Ann Behav Med 2014. 